\title{
A 3-DIMENSIONAL HYPERBOLIC STEFAN PROBLEM WITH DISCONTINUOUS TEMPERATURE
}

\author{
By \\ DENING LI \\ West Virginia University
}

\begin{abstract}
The hyperbolic heat transfer model is obtained by replacing the classical Fourier's law with the relaxation relation $\tau \mathbf{q}_{t}+\mathbf{q}=-k \nabla T$. The conditions are derived for the local existence and uniqueness of classical solutions for a 3-dimensional Stefan problem of hyperbolic heat transfer model where the temperature may sustain a jump across the phase change interface.
\end{abstract}

1. Introduction. In this paper, we shall consider the phase-change model proposed by J. Greenberg in [4] for the hyperbolic heat transfer model (see also [15]). The new feature of the model is that instead of the usual assumption on the continuity of the temperature across the phase-change interface, it is assumed that the relaxation relation between the temperature gradient $\nabla T$ and the heat flux $\mathbf{q}$ be interpreted as a conservation law that should also be satisfied across the interface. Based upon this assumption, a hyperbolic Stefan problem in one-dimensional space was discussed in [4]. In this paper, the 3-dimensional case will be considered by applying the theory of energy estimate for multi-dimensional hyperbolic boundary value problems.

The classical mathematical model for heat transfer and diffusion phenomena is of parabolic type, based upon Fourier's law

$$
\mathbf{q}=-k \nabla T .
$$

Here $\mathbf{q}=\left(q_{1}, q_{2}, q_{3}\right)$ is the heat flux vector, $T$ the temperature, and $k$ the thermal conductivity. However, in a number of situations, this model is unsatisfactory, especially because it might imply an infinite speed of propagation. Various modifications to this model have been suggested (see [5] for a recent survey). One of the most popular choices to modify this model is to assume that the heat flux $\mathbf{q}$ responds to temperature gradient $\nabla T$ not instantly but over a period of time (see, e.g., [13]):

$$
\mathbf{q}=-\int_{0}^{\infty} a(s) \nabla T(t-s) d s .
$$

In particular, for $a(s)=(k / \tau) \exp (-s / \tau),(1.2)$ becomes

$$
\tau \partial_{t} \mathbf{q}+\mathbf{q}=-k \nabla T
$$

Received July 6, 1990.

1980 Mathematics Subject Classification (1985 Revision). Primary 35L70, 80A20; Secondary 35L20.

Key words and phrases. Hyperbolic system, heat transfer, Stefan problem. 
where the relaxation time $\tau$ reflects the length of the relaxation period. The hyperbolic heat transfer model is obtained by replacing the Fourier's law (1.1) with the relaxation relation (1.3). In addition to (1.3), we have the conservation of heat energy

$$
\partial_{t} e+\nabla \cdot \mathbf{q}=0
$$

where the internal energy $e$ is determined by

$$
e= \begin{cases}c T, & T<0, \\ L+c T, & T>0,\end{cases}
$$

with $c$ being the specific heat and $L$ the latent heat.

The Stefan free boundary problem discussed here for the hyperbolic system (1.3), (1.4) is different from the usual Stefan type problems in $[7,13,14]$ in that $(1.3),(1.4)$ will be understood as conservation laws. Consequently, the free boundary conditions imposed upon the phase change interface should be the Rankine-Hugoniot conditions, usually imposed in the discussion of free boundary problems for conservation laws.

Assume the phase-change interface be described locally by

$$
x_{3}=\phi\left(x^{\prime}, t\right), \quad x^{\prime}=\left(x_{1}, x_{2}\right) .
$$

Then the free boundary conditions on (1.6) corresponding to (1.3), (1.4) are

$$
\left\{\begin{array}{l}
{\left[\tau q_{1}\right] \phi_{t}+[k T] \phi_{x_{1}}=0} \\
{\left[\tau q_{2}\right] \phi_{t}+[k T] \phi_{x_{2}}=0} \\
{\left[\tau q_{3}\right] \phi_{t}-[k T]=0} \\
{[(L+c T)] \phi_{t}+\left[q_{1}\right] \phi_{x_{1}}+\left[q_{2}\right] \phi_{x_{2}}-\left[q_{3}\right]=0}
\end{array}\right.
$$

or briefly denoted as

$$
\mathscr{B}(\mathbf{q}, T, \phi)=0 \text {. }
$$

Here, $[f]$ denotes, as usual, the jump difference of $f$ across the interface (1.6). In particular, the adoption of (1.7) implies that discontinuous temperature across the phase change interface would be acceptable, as in gas dynamics.

Since the energy function (1.5) is nonconvex, one cannot expect the two-phase problem to be well-posed even in the 1-dimensional case [4]. Hence we will restrict ourselves in the following to the discussion of the one-phase problem.

Now, for the hyperbolic system (1.3), (1.4), we have the discontinuous initial data

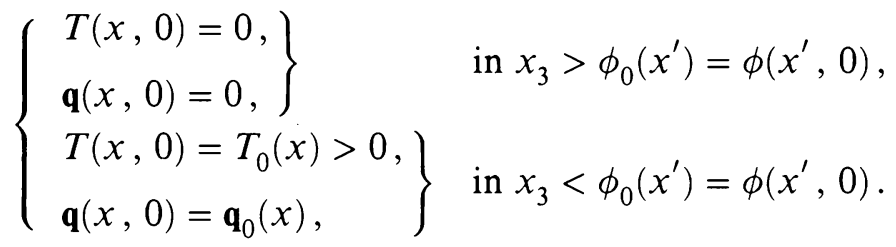

Denoting $u \equiv\left(q_{1}, q_{2}, q_{3}, T\right)^{t}$, we can write (1.3), (1.4) as the hyperbolic system

$$
\mathscr{L} u \equiv \partial_{t} u+\sum_{j=1}^{3} A_{j} \partial_{x_{j}} u+B u=0
$$


where

$$
\begin{array}{rlrl}
A_{1}= & \left(\begin{array}{cccc}
0 & 0 & 0 & k / \tau \\
0 & 0 & 0 & 0 \\
0 & 0 & 0 & 0 \\
1 / c & 0 & 0 & 0
\end{array}\right), & A_{2}=\left(\begin{array}{cccc}
0 & 0 & 0 & 0 \\
0 & 0 & 0 & k / \tau \\
0 & 0 & 0 & 0 \\
0 & 1 / c & 0 & 0
\end{array}\right), \\
A_{3}=\left(\begin{array}{cccc}
0 & 0 & 0 & 0 \\
0 & 0 & 0 & 0 \\
0 & 0 & 0 & k / \tau \\
0 & 0 & 1 / c & 0
\end{array}\right), & B=\left(\begin{array}{cccc}
1 / \tau & 0 & 0 & 0 \\
0 & 1 / \tau & 0 & 0 \\
0 & 0 & 1 / \tau & 0 \\
0 & 0 & 0 & 0
\end{array}\right) .
\end{array}
$$

We notice here that the eigenvalues of $\Sigma A_{j} \omega_{j}$ are $\lambda_{1}=\lambda_{2}=0, \lambda_{3}, \lambda_{4}= \pm \alpha|\omega|$ with the propagation speed $\alpha=\sqrt{\frac{k}{\tau c}}$ and $|\omega|^{2}=\sum \omega_{j}^{2}$. In particular, we remark that the 2-dimensional version of the problem is strictly hyperbolic system and the 3-dimensional system (1.9) satisfies the block structure assumption in [10, 11].

In order to obtain classical solutions to the problem (1.7)-(1.9), certain compatibility conditions are necessary. These are conditions imposed upon the traces of the initial data $T_{0}, \mathbf{q}$ at $x_{3}=\phi_{0}\left(x^{\prime}\right)$. They could be explicitly obtained by comparing the two values of $u_{t}(x, 0)$ at $x_{3}=\phi_{0}\left(x^{\prime}\right)$, one computed from the interior equations (1.9) and another from the free boundary condition (1.7). Higher-order compatibility conditions can be formulated similarly. We will omit the details here, the interested reader could refer to, e.g., [11, 12].

An equivalent way to state the compatibility condition is the following: The initial data in (1.7)-(1.9) are said to satisfy $k$ th order compatibility conditions if there exist approximate solutions $(\tilde{u}, \tilde{\phi}) \in C^{\infty}$ of $(1.7)-(1.9)$ such that

$$
\left\{\begin{array}{l}
\tilde{u}(x, 0)=u_{0}(x), \quad \tilde{\phi}\left(x^{\prime}, 0\right)=\phi_{0}\left(x^{\prime}\right), \\
\mathscr{L} \tilde{u}=O\left(t^{k}\right), \\
\mathscr{B}(\tilde{u}, \tilde{\phi})=O\left(t^{k}\right) .
\end{array}\right.
$$

Now the main result of this paper can be stated in the following

THEOREM 1.1. In the one-phase hyperbolic Stefan problem (1.7)-(1.9), assuming that

1. $u_{0}(x), \phi_{0}\left(x^{\prime}\right) \in C^{\infty}$;

2. $k$ th order compatibility conditions (1.10) are satisfied with $k \geq 4$;

3. the following stability condition is satisfied:

$$
\tilde{\phi}_{t}\left(x^{\prime}, 0\right)<\alpha \text {, }
$$

there is $t_{0}>0$ such that in $\left[0, t_{0}\right),(1.7)-(1.9)$ has a unique classical solution $(u, \varphi) \in H^{k}\left(\left(0, t_{0}\right) \times R^{3}\right) \times H^{k+1}\left(\left(0, t_{0}\right) \times R^{2}\right)$.

REMARK 1.1. The stability condition (1.11) is a natural requirement, because of the fact that $\alpha$ is the propagation speed. Therefore, (1.11) simply means that the initial data should be such that the initial speed of the moving free boundary should be smaller than the propagation speed.

REMARK 1.2. The problem (1.7)-(1.9) discussed here is in fact a little different from the 1-dimensional problem considered in [4]. In [4], the problem is proposed with temperature $T$ given to be constant on a fixed boundary and without initial data 
sirce the initial position of the free boundary coincides with the fixed boundary. The exact 3-dimensional counterpart of the problem in [4] can also be treated by blowing up the corner domain and assuming higher-order compatibility conditions. The proof proceeds as in this paper and employing the technique in [2]. We will not go into the details here.

REMARK 1.3. The general $n$-dimensional case can be discussed exactly the same way. One needs only to replace the condition $k \geq 4$ in Theorem 1.1 by $k \geq$ $(n+1) / 2+2$.

\section{Transformation.}

2.1. Fix free boundary. First of all, we introduce the new coordinates $(s, y)$, depending upon the unknown interface of phase change, such that in the new coordinates, the original free boundary becomes the fixed boundary $y_{3}=0$. Let

$$
t=s, \quad x^{\prime}=y^{\prime}, \quad x_{3}-\phi\left(x^{\prime}, t\right)=y_{3} .
$$

Then in the new coordinates, if denoted again by $(t, x)$, Eq. (1.9) becomes

$$
\mathscr{L}_{1}(\phi) u=0 \text { in } x_{3}<0,
$$

where

$$
\mathscr{L}_{1}(\phi) \equiv \partial_{t}+\sum_{j=1}^{2} A_{j} \partial_{x_{j}}+\left(A_{3}-\phi_{t}-\sum_{j=1}^{2} A_{j} \phi_{x_{j}}\right) \partial_{x_{3}}+B .
$$

The boundary condition (1.7) remains the same:

$$
\begin{cases}\tau q_{1} \phi_{t}+k T \phi_{x_{1}}=0, & \\ \tau q_{2} \phi_{t}+k T \phi_{x_{2}}=0, & \text { on } x_{3}=0 . \\ \tau q_{3} \phi_{t}-k T=0, & \\ (L+c T) \phi_{t}+q_{1} \phi_{x_{1}}+q_{2} \phi_{x_{2}}-q_{3}=0, & \end{cases}
$$

Without loss of generality, we may assume in (1.8) that

$$
\phi_{0}(0)=0, \quad \nabla \phi_{0}(0)=0 .
$$

Because of the discontinuous temperature assumption $T>0$ and $\phi_{t} \geq 0$ on the phase change interface, at $t=0, x^{\prime}=0$ we deduce from (2.3) that

$$
\phi_{t}(0,0)>0, \quad q_{1}(0,0)=q_{2}(0,0)=0 .
$$

Consequently, from (2.3), at $t=0, x^{\prime}=0$ the initial data satisfy

$$
q_{3}^{2}=\frac{k T}{\tau}(L+c T)=\alpha^{2} c T(L+c T) .
$$

2.2. Homogeneous initial data. From the compatibility assumption (1.10), we introduce new unknown variables $(v, \psi)$ to reduce the initial data in (1.8) to be zeros. Let

$$
u \equiv \tilde{u}+v, \quad \phi \equiv \tilde{\phi}+\psi
$$


Substituting the value $(u, \phi)$ in (2.6) into (2.2), (2.3), we obtain for the new unknown variables $(v, \psi)$ the following boundary value problem with homogeneous initial data:

$$
\begin{aligned}
& \mathscr{L}_{2}(v, \psi)(v, \psi) \equiv \mathscr{L}_{1}(\tilde{\phi}+\psi)(\tilde{u}+v)-\mathscr{L}_{1}(\tilde{\phi})(\tilde{u}) \\
&=\mathscr{L}_{1}(\tilde{\phi}+\psi) v-\partial_{x_{3}} \tilde{u}\left(\partial_{t}+\sum_{j=1}^{2} A_{j}\left(\tilde{\partial}_{x_{j}}\right)\right) \psi f, \quad \text { in } x_{3}>0, \\
& \mathscr{B}_{2}(v, \psi)(v, \psi) \equiv \mathscr{B}(\tilde{u}+v, \tilde{\phi}+\psi)-\mathscr{B}(\tilde{u}, \tilde{\phi})=g .
\end{aligned}
$$

Here, $(f, g)$ are smooth functions which have zero traces at $t=0$ up to order $k$.

Obviously, the solution of the problem (1.7)-(1.9) is equivalent to the solution of (2.7)-(2.8). Therefore, in order to prove our main theorem, we need only to prove the corresponding result of existence and uniqueness for (2.7), (2.8). This will be done by discussing the well-posed condition for the linearized problem of $(2.7),(2.8)$ and by linear iteration.

3. Linearized problem. In this section, we linearize the problem (2.7), (2.8) and discuss the well-posedness of the linearized problem. The linearization about $(\tilde{u}, \tilde{\phi})$ to be used in the next section is the following:

$$
\begin{gathered}
\partial_{t} v+\sum_{j=1}^{3} \tilde{A}_{j} \partial_{x_{j}} v+\widetilde{B} v+\widetilde{C} \partial \psi=f, \\
b\left(\partial_{t}, \partial_{x^{\prime}}\right) \psi+P v=g .
\end{gathered}
$$

In particular, at $(0,0)$ the coefficients in $(3.1),(3.2)$ become

$$
\tilde{A}_{j}=A_{j}, \quad j=1,2, \quad \tilde{A}_{3}=-\mu I+A_{3},
$$

with $\mu=\tilde{\phi}_{t}(0,0)$ and

$$
\begin{gathered}
b\left(\partial_{t}, \partial_{x^{\prime}}\right)=\left(\begin{array}{c}
k T \partial_{x_{1}} \\
k T \partial_{x_{2}} \\
\tau q_{3} \partial_{t} \\
(L+c T) \partial_{t}
\end{array}\right), \\
P=\left(\begin{array}{cccc}
\tau \mu & 0 & 0 & 0 \\
0 & \tau \mu & 0 & 0 \\
0 & 0 & \tau \mu & -k \\
0 & 0 & -1 & c \mu
\end{array}\right) .
\end{gathered}
$$

To discuss the linear problem (3.1), (3.2), we introduce the usual hyperbolic $\eta$ weighted interior norm

$$
\|u\|_{s, \eta}^{2}=\sum_{|\alpha| \leq s} \int_{R^{3} \times R_{+}^{1}}\left|\partial_{t, x}^{\alpha}\left(e^{-\eta t} u\right)\right|^{2} d x d t
$$

and the boundary norm

$$
|u|_{s, \eta}^{2}=\sum_{|\alpha| \leq s} \int_{R^{3}}\left|\partial_{t, x^{\prime}}^{\alpha}\left(e^{-\eta t} u\right)\right|^{2} d x^{\prime} d t
$$


The corresponding inner products will be denoted by $(\cdot, \cdot)_{s, \eta}$ and $\langle\cdot, \cdot\rangle_{s, \eta}$, respectively.

As in [10], the linear boundary value problem for $(v, \psi)$ is called uniformly stable if the solution $(v, \psi)$ satisfies the estimate

$$
\eta\|v\|_{0, \eta}^{2}+|v|_{0, \eta}^{2}+|\psi|_{1, \eta}^{2} \leq C\left(\frac{1}{\eta}\|f\|_{0, \eta}+|g|_{0, \eta}\right) \text { for } \eta \geq \eta_{0} .
$$

The main result of this section is the following

THEOREM 3.1. Under the assumption of Theorem 1.1 upon the initial data, smooth solutions of the linearized problem $(3.1),(3.2)$ satisfy the estimate $(3.6)$ near $(0,0)$.

Proof. Since $T>0$ on $x_{3}<0$, so $|b(s, i \xi)|>0$ by (3.4) for all $|s|^{2}+|\xi|^{2}=1$ with $s=\eta+i \tau$.

Now define the projector $\Pi(s, i \xi)$ in $C^{4}$ as in [10]:

$$
\Pi(x, i \xi) w \equiv b(s, i \xi)-\frac{(b(s, i \xi), w)}{|b(s, i \xi)|^{2}} b(s, i \xi) .
$$

Applying the pseudo-differential operator $\Pi\left(\eta+\partial_{t}, \partial_{x^{\prime}}\right)$ to the boundary condition (3.2) and omitting the lower-order terms, we obtain

$$
\Pi\left(\eta+\partial_{t}, \partial_{x^{\prime}}\right) P v=-(\Pi b)\left(\eta+\partial_{t}, \partial_{x^{\prime}}\right) \psi+\Pi\left(\eta+\partial_{t}, \partial_{x^{\prime}}\right) g \equiv g_{1} .
$$

Rewrite (3.1) as

$$
\partial_{x_{3}} v+\tilde{A}_{3}^{-1}\left(\partial_{t}+\sum_{j=1}^{2} \tilde{A}_{j} \partial_{x_{j}}+\widetilde{B}\right) v+\tilde{A}_{3}^{-1} \tilde{C} \partial \psi=\tilde{A}_{3}^{-1} f .
$$

Let

$$
M(s, i \xi) \equiv \tilde{A}_{3}^{-1}\left(s+\sum_{j=1}^{2} i \tilde{A}_{j} \xi_{j}\right)
$$

then the proof of Theorem 3.1 can be derived from the following proposition.

Proposition 3.1. Under the assumption of Theorem 3.1, there exists a symmetric matrix $\mathscr{R}(s, i \xi)$ such that

1. $\operatorname{Re}(\mathscr{R} M)(s, i \xi) \geq \delta \eta$ on $|s|^{2}+|\xi|^{2}=1, \operatorname{Re} s=\eta \geq 0$;

2. $\mathscr{R}(s, i \xi)+\delta_{*}^{-1}(\Pi P)^{*}(\Pi P)(s, i \xi) \geq \delta_{*} I$,

where constants $\delta, \delta_{*}$ depend only upon the $C^{0}$ norms of the coefficients, or by the imbedding theorem, depend only upon the local $\mathrm{H}^{3}$ norms of the coefficients.

We postpone the proof of Proposition 3.1 to Sec. 5. Now, assuming Proposition 3.1, we can prove Theorem 3.1 as follows.

Applying the operator $\mathscr{R}\left(\eta+\partial_{t}, \partial_{x^{\prime}}\right)$ to (3.9) and taking the inner product with $v$ in the $\eta$-weighted space $L^{2}\left(R^{+} \times R^{3}\right)$, we have

$$
\left(v, \mathscr{R} M\left(\eta+\partial_{t}, \partial_{x^{\prime}}\right) v\right)_{0, \eta}=-\left(v, \mathscr{R} \partial_{x_{3}} v\right)_{0, \eta}+\left(v, \tilde{A}_{3}^{-1}(\widetilde{B} v+\widetilde{C} \partial \psi-f)\right)_{0, \eta} \text {. }
$$

By Proposition 3.1, we can apply the sharp Gårding's inequality to obtain

$$
\operatorname{Re}\left(v, \mathscr{R} M\left(\eta+\partial_{t}, \partial_{x^{\prime}}\right) v\right)_{0, \eta} \geq \frac{\delta \eta}{2}\|v\|_{0, \eta}^{2},
$$


with $\eta \geq \eta_{0} \gg 1$. By the result on $L^{2}$-boundedness of pseudo-differential operators with $H^{s}$ coefficients $[1,6], \eta_{0}$ depends only upon the local $H^{4}$ norms of the coefficients.

Integrating by parts and noticing that $\mathscr{R}$ is symmetric (hence $\mathscr{R}-\mathscr{R}^{*}$ is an operator of order -1 ), and also employing the fact that the matrix $\widetilde{A}_{3}$ is nonsingular, we have

$$
\left(v, \mathscr{R} \partial_{x_{3}} v\right)_{0, \eta} \geq\langle v, \mathscr{R} v\rangle_{0, \eta}-C\left(\|v\|_{0, \eta}^{2}+|\psi|_{0, \eta}^{2}\right) .
$$

Again, by Proposition 3.1 and the sharp Gårding inequality we obtain

$$
\left(v, \mathscr{R} \partial_{x_{3}} v\right)_{0, \eta} \geq \frac{\delta_{*}}{2}|v|_{0, \eta}^{2}-C|\Pi P v|_{0, \eta}^{2} \geq \frac{\delta_{*}}{2}|v|_{0, \eta}^{2}-C\left(|g|_{0, \eta}^{2}+|\psi|_{0, \eta}^{2}\right) \text {. }
$$

Here, we have used the fact that the operator $\Pi b$ is of order zero. Again, the constants in (3.13) depend only upon the local $H^{4}$ norms of the coefficients.

Combining (3.10)-(3.13), we obtain

$$
\eta\|v\|_{0, \eta}^{2}+|v|_{0, \eta}^{2} \leq C\left(\frac{1}{\eta}\left(\|f\|_{0, \eta}^{2}+|\psi|_{1, \eta}^{2}\right)+|g|_{0, \eta}^{2}\right),
$$

with $\eta \geq \eta_{0} \gg 1$.

Since $b(s, i \xi) \neq 0$ on $|s|^{2}+|\xi|^{2}=1$, from the overdetermined system (3.2) we deduce

$$
|\psi|_{1, \psi} \leq C\left(|v|_{0, \eta}+|g|_{0, \eta}\right) .
$$

Theorem 3.1 follows readily from (3.14), (3.15).

Following a similar procedure as in the proof of Theorem 3.1, we can obtain the high-order estimate for the problem (3.1), (3.2):

THEOREM 3.2. Under the assumption of Theorem 1.1 upon the initial data, for any positive integer $k$, smooth solutions of the linearized problem $(3.1),(3.2)$ satisfy the following estimate $(3.16)$ near $(0,0)$ :

$$
\eta\|v\|_{k, \eta}^{2}+|v|_{k, \eta}^{2}+|\psi|_{k+1, \eta}^{2} \leq C_{k}\left(\frac{1}{\eta}\|f\|_{k, \eta}^{2}+|g|_{k, \eta}^{2}\right) \text { for } \eta \geq \eta_{0},
$$

where the constant $C_{k}$ depends only upon the local $H^{s}$ norms of the coefficients, with $s=\max \{k, 4\}$.

The theorem can be proved in a standard manner. First, take the tangential derivatives of the problem (3.1), (3.2) and derive the high order tangential estimates for $(v, \psi)$. Then make use of the noncharacteristic boundary to obtain high order normal derivatives. The dependency of the constant upon the coefficients is obtained by employing the Nirenberg-Gagliardo inequality

$$
\|u v\|_{k} \leq C\left(\|u\|_{3}\|v\|_{k}+\|u\|_{k}\|v\|_{3}\right) .
$$

Finally, since the formal adjoint of a boundary value problem satisfies the uniform Lopatinski condition if the original problem does, from the energy estimate (3.16) and the standard dual argument and Riesz representation theorem, we have 
the following existence result:

THEOREM 3.3. If the problem (3.1), (3.2) is uniformly stable, then for any $k \geq 0$ there is a unique solution $(v, \psi) \in H^{k} \times H^{k+1}$ satisfying (3.16).

Here, the uniqueness comes readily from the energy estimate.

4. Nonlinear problem. In this section we shall use the iteration method to show the existence of a unique classical solution $(v, \psi)$ of (2.7), (2.8) in the neighborhood of $(x, t)=(0,0)$, or equivalently the existence of the unique classical solution $(u, \phi)$ of (1.7)-(1.9). Since in the estimate (3.16) for the linearized problem the estimate of boundary function $\psi$ is one order higher than the estimate of $v$, we do not have to employ the quadratic error in newtonian iteration. Therefore we can use classical linear iteration to solve (2.7), (2.8).

For the nonlinear boundary value problem $(2.7),(2.8)$ of $(v, \psi)$, renumbered as

$$
\begin{cases}\mathscr{L}_{2}(v, \psi)(v, \psi)=f & \text { in } x_{3}<0, \\ \mathscr{B}_{2}(v, \psi)(v, \psi)=g & \text { on } x_{3}=0,\end{cases}
$$

we have the following

THEOREM 4.1. Suppose that in the problem (4.1), for $k \geq 4$,

1. $(f, g) \in H^{k}$ with zero traces at $t=0$ up to the order $k-1$;

2. at $(v, \psi)=(0,0)$, the boundary value problem is uniformly stable, i.e., the estimate (3.6) is satisfied.

Then there is $t_{0}>0$ such that in $\left(0, t_{0}\right)$, problem (4.1) has a unique solution $(v, \psi) \in H^{k} \times H^{k+1}$. Moreover,

$$
\eta\|v\|_{k, \eta, t_{0}}^{2}+|v|_{k, \eta, t_{0}}^{2}+|\psi|_{k+1, \eta, t_{0}}^{2} \leq C_{k}\left(\frac{1}{\eta}\|f\|_{k, \eta, t_{0}}^{2}+|g|_{k, \eta, t_{0}}^{2}\right) \quad \text { for } \eta \geq \eta_{0} .
$$

Here the norms $\|\cdot\|_{s, \eta, t_{0}},|\cdot|_{s, \eta, t_{0}}$ denote, respectively, the restriction in $\left[0, t_{0}\right]$ of the norms $\|\cdot\|_{s, \eta},|\cdot|_{s, \eta}$.

Proof. Let $\chi_{r}$ be a smooth cutoff function of the interval $[0, r]$,

$$
\chi_{r}(t)=\left\{\begin{array}{l}
1, \quad 0 \leq t \leq r, \\
\text { smooth and monotone decreasing, } \quad r \leq t \leq 2 r, \\
0, \quad 2 r \leq t .
\end{array}\right.
$$

Denote $\tilde{\phi}_{r}=\chi_{r} \tilde{\phi}+\left(1-\chi_{r}\right) t \tilde{\phi}^{\prime}(0), \tilde{u}_{r}=\chi_{r} \tilde{u}+\left(1-\chi_{r}\right) \tilde{u}(0)$, and $\left(f_{r}, g_{r}\right)=\chi_{r}(f, g)$. Then consider the cutoff problem of (4.1):

$$
\begin{cases}\mathscr{L}_{r}(v, \psi)(v, \psi) \equiv \mathscr{L}_{1}\left(\tilde{u}_{r}+v, \tilde{\phi}_{r}+\psi\right)-\mathscr{L}_{1}\left(\tilde{\phi}_{r}\right)\left(\tilde{u}_{r}\right)=f_{r} & \text { in } x_{3}<0, \\ \mathscr{B}_{r}(v, \psi)(v, \psi) \equiv \mathscr{B}\left(\tilde{u}_{r}+v, \tilde{\phi}_{r}+\psi\right)-\mathscr{B}\left(\tilde{u}_{r}, \tilde{\phi}_{r}\right)=g_{r} & \text { on } x_{3}=0 .\end{cases}
$$

Obviously, the proof of Theorem 4.1 is equivalent to proving the unique existence of a $H^{k} \times H^{k+1}$ solution $(v, \psi)$ for (4.3).

Let $\left(v_{0}, \psi_{0}\right)=(0,0)$ and, for all $j>0$, let $\left(v_{j}, \psi_{j}\right)$ be the solution of the following linearized boundary value problem:

$$
\left\{\begin{array}{ll}
\mathscr{L}_{r}\left(v_{j-1}, \psi_{j-1}\right)\left(v_{j}, \psi_{j}\right)=f_{r} & \text { in } x_{3}<0, \\
\mathscr{B}_{r}\left(v_{j-1}, \psi_{j-1}\right)\left(v_{j}, \psi_{j}\right)=g_{r} & \text { on } x_{3}=0,
\end{array} \quad j>0 .\right.
$$


We want to show that for small $r,(4.4)$ is uniformly stable for all $j>0$ and hence solvable. Moreover, the solution sequence $\left(v_{j}, \psi_{j}\right)$ of $(4.4)$ converges to a solution of (4.3).

Noticing that the uniform stability of the linear boundary problem (4.4) depends continuously upon the coefficients [6] and (4.4) is uniformly stable at $\left(v_{j-1}, \psi_{j-1}\right)=$ $(0,0)$, we see that $(4.4)$ will be uniformly stable for all $\max \left|v_{j-1}(x, t)\right|+\left|\psi_{j-1}\left(x^{\prime}, t\right)\right|$ $\ll 1$. By the Sobolev imbedding theorem, we conclude that there is a constant $\varepsilon_{0}$ such that (4.4) is well-posed for all

$$
\left\|v_{j-1}\right\|_{k, \eta}^{2}+\left|v_{j-1}\right|_{k, \eta}^{2}+\left|\psi_{j-1}\right|_{k+1, \eta}^{2} \leq \varepsilon_{0}
$$

where $k \geq 4$. Moreover, from the dependency argument of the constant $C_{k}$ in the energy estimate (3.16), we see that $C_{k}$ depends only upon $\varepsilon_{0}$. Therefore in order to show the solvability of (4.4), we need only show that (4.5) is true for all $j>0$.

By induction, let $(4.5)$ be true for $j-1$. Then from Theorems 3.2-3.3, (4.4) has a unique solution $\left(v_{j}, \psi_{j}\right)$ satisfying

$$
\eta\left\|v_{j}\right\|_{k, \eta}^{2}+\left|v_{j}\right|_{k, \eta}^{2}+\left|\psi_{j}\right|_{k+1, \eta}^{2} \leq C_{k}\left(\frac{1}{\eta}\left\|f_{r}\right\|_{k, \eta}+\left|g_{r}\right|_{k, \eta}\right) .
$$

Because $(f, g)$ has zero traces at $t=0$ up to order $k-1,\left\|f_{r}\right\|_{k, \eta}+\left|g_{r}\right|_{k, \eta} \rightarrow 0$ as $r \rightarrow 0$. So for fixed $\eta$ and $\varepsilon_{0}$ (consequently fixed $C_{k}$ ), choosing $r$ sufficiently small, we can make the right side of (4.6) smaller than $\varepsilon_{0}$. Hence (4.5) is valid for all $j>0$.

It remains to show that the sequence $\left(v_{j}, \psi_{j}\right)$ converges strongly in the appropriate norms. We have:

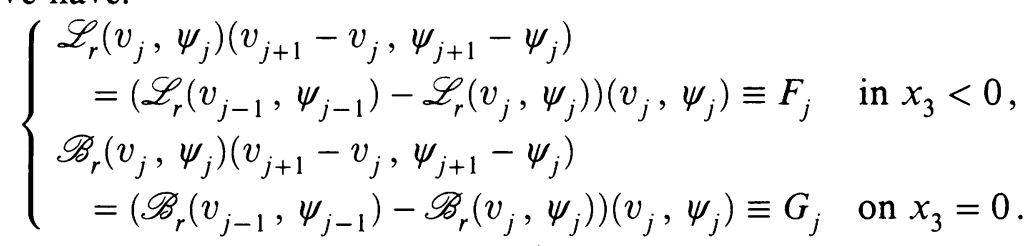

We now estimate $F_{j}$ and $G_{j}$. Since $H^{k-1}$ is an algebra, we have

$$
\begin{aligned}
\left\|F_{j}\right\|_{k-1, \eta}^{2}+\left|G_{j}\right|_{k-1, \eta}^{2} \leq & C\left\{\left\|v_{j}\right\|_{k, \eta}^{2}+\left|v_{j}\right|_{k, \eta}^{2}+\left|\psi_{j}\right|_{k+1, \eta}^{2}\right\} \\
& \cdot\left\{\left\|v_{j}-v_{j-1}\right\|_{k-1, \eta}^{2}+\left|v_{j}-v_{j-1}\right|_{k-1, \eta}^{2}+\left|\psi_{j}-\psi_{j-1}\right|_{k, \eta}^{2}\right\} \\
\leq & C \varepsilon_{0}\left\{\left\|v_{j}-v_{j-1}\right\|_{k-1, \eta}^{2}+\left|v_{j}-v_{j-1}\right|_{k-1, \eta}^{2}+\left|\psi_{j}-\psi_{j-1}\right|_{k, \eta}^{2}\right\} .
\end{aligned}
$$

Applying the estimate (3.16) and choosing $\varepsilon_{0}$ sufficiently small, we have

$$
\begin{aligned}
& \left\|v_{j+1}-v_{j}\right\|_{k-1, \eta}^{2}+\left|v_{j+1}-v_{j}\right|_{k-1, \eta}^{2}+\left|\psi_{j+1}-\psi_{j}\right|_{k, \eta}^{2} \\
& \quad \leq \frac{1}{2}\left\{\left\|v_{j}-v_{j-1}\right\|_{k-1, \eta}^{2}+\left|v_{j}-v_{j-1}\right|_{k-1, \eta}^{2}+\left|\psi_{j}-\psi_{j-1}\right|_{k, \eta}^{2}\right\} .
\end{aligned}
$$

This implies $\left(v_{j}, \psi_{j}\right)$ converges in $H^{k-1} \times H^{k}$ to $(v, \psi)$. Since the norms of $\left(v_{j}, \psi_{j}\right)$ in $H^{k} \times H^{k+1}$ are uniformly bounded, $(v, \psi) \in H^{k} \times H^{k+1}$ by the BanachSaks theorem. 
It is not difficult to check that $(v, \psi)$ is the unique solution of the problem, following the same procedure leading to (4.9). Thus the proof of Theorem 4.1 is complete.

5. Proof of Proposition 3.1. In this section we shall show that under the assumption of Theorem 3.1, the matrix $\mathscr{R}(s, i \xi)$ in Proposition 3.1 exists. First of all, from the microlocal structure of $\mathscr{R}$, we need only show the matrix $\mathscr{R}$ exists for a fixed coefficient at $(x, t)=(0,0)$. That is, we should consider the following problem with the constant coefficient

$$
\begin{cases}\partial_{x_{3}} v+M\left(\partial_{t}, \partial_{x^{\prime}}\right) v=f & \text { in } x_{3}<0, \\ \Pi P v=g & \text { on } x_{3}=0,\end{cases}
$$

with the matrix $M(s, i \xi)$ defined as

$$
M(s, i \xi)=\left(-\mu+A_{3}\right)^{-1}\left(s+i A_{1} \xi_{1}+i A_{2} \xi_{2}\right) .
$$

From (1.9) and noticing $\alpha^{2}=k / \tau c$, we have

$$
\begin{aligned}
M(s, i \xi) & =\left(\begin{array}{cccc}
-\mu & & \\
& -\mu & & \\
& -\mu & k / \tau \\
& 1 / c & -\mu
\end{array}\right)^{-1}\left(\begin{array}{cccc}
s & & \begin{array}{c}
i \xi_{1} k / \tau \\
i \xi_{2} k / \tau \\
\end{array} \\
& s & s & 0 \\
i \xi_{1} / c & i \xi_{2} / c & 0 & s
\end{array}\right) \\
& =\left(\begin{array}{ccccc}
\frac{-s}{\mu} & 0 & 0 & \frac{-i \xi_{1} k}{\tau \mu} \\
0 & \frac{-s}{\mu} & 0 & \frac{i \xi_{2} k}{\tau \mu} \\
\frac{i \xi_{1} \alpha^{2}}{\alpha^{2}-\mu^{2}} & \frac{i \xi_{2} \alpha^{2}}{\alpha^{2}-\mu^{2}} & \frac{s \mu}{\alpha^{2}-\mu^{2}} & \frac{s k}{\tau\left(\alpha^{2}-\mu^{2}\right)} \\
\frac{i \xi_{1} \mu}{c\left(\alpha^{2}-\mu^{2}\right)} & \frac{i \xi_{2} \mu}{c\left(\alpha^{2}-\mu^{2}\right)} & \frac{s}{c\left(\alpha^{2}-\mu^{2}\right)} & \frac{s \mu}{\alpha^{2}-\mu^{2}}
\end{array}\right) .
\end{aligned}
$$

For the problem (5.1), it was shown in [6] that the existence of the symmetrizer $\mathscr{R}$ can be reduced to an algebraic criterion, namely, we have the following

Proposition 5.1. For $\operatorname{Re} s=\eta>0$, the eigenvalues of $M(s, i \xi)$ have nonzero real parts. Let $\mathscr{E}^{+}(s, i \xi)$ denote the generalized eigenspace spanned by all the generalized eigenvectors corresponding to the eigenvalues with positive real part. Then the symmetrizer matrix $\mathscr{R}(s, i \xi)$ exists if

1. $\operatorname{dim} \mathscr{E}^{+}=3$;

2. the matrix $\Pi P(s, i \xi)$ is nonsingular on $\mathscr{E}^{+}(s, i \xi)$, uniformly with respect to $\eta>0$.

Therefore, in order to prove Proposition 3.1, we need only check the two conditions in Proposition 5.1. First, we compute the eigenvalues $\lambda$ of $M(s, i \xi)$. Direct calculation gives

$$
\left\{\begin{array}{l}
\lambda_{1}=\lambda_{2}=\frac{s}{\mu}, \\
\lambda_{3,4}=\frac{-\mu s \pm \sqrt{s^{2}+|\xi|^{2}\left(\alpha^{2}-\mu^{2}\right)}}{\alpha^{2}-\mu^{2}} .
\end{array}\right.
$$


Under the assumption of Proposition 3.1, $\alpha^{2}-\mu^{2}>0$. Therefore, it is readily checked that $\operatorname{Re} \lambda_{1}, \operatorname{Re} \lambda_{2}, \operatorname{Re} \lambda_{3}>0, \operatorname{Re} \lambda_{4}<0$.

To check the second condition in Proposition 5.1, we write the Fourier-Laplace transformation of (3.2) as

$$
\left\{\begin{array}{l}
i \xi_{1} k T \psi+\tau \mu v_{1}=0, \\
i \xi_{2} k T \psi+\tau \mu v_{2}=0, \\
s \tau q_{3} \psi+\tau \mu v_{3}-k v_{4}=0, \\
s(L+c T) \psi-v_{3}+c \mu v_{4}=0 .
\end{array}\right.
$$

Here, by (2.3), (2.5), we have the relations between the parameters

$$
\tau q_{3} \mu=k T, \quad(L+c T) \mu=q_{3} .
$$

We should, for each point $(s, i \xi)$ on $|s|^{2}+|\xi|^{2}=1$ and $\operatorname{Re} s=\eta>0$,

1. eliminate the unknown function $\psi$ from (5.5),

2. apply the resulting operator for $v$ to the three eigenvectors (or generalized eigenvectors) consisting of a base of $\mathscr{E}^{+}$.

Then the second condition in Proposition 5.1 is valid if we end up with three linearly independent vectors. In order to obtain the uniform independence of the vectors, we need to check the limit case as $\eta \rightarrow 0$.

First we notice that $\lambda_{1}=\lambda_{2}=\lambda_{3}$ if and only if $s=\mu|\xi|$, here $|\xi|^{2}=\xi_{1}^{2}+\xi_{2}^{2}$. We shall consider three cases.

Case I: $s \neq \mu|\xi|$ and $|\xi| \neq 0$. In this case, we have for the eigenvalues $\lambda_{1}=\lambda_{2}$ two independent eigenvectors

$$
v^{(1)}=\left(\begin{array}{c}
\xi_{2} \\
-\xi_{1} \\
0 \\
0
\end{array}\right), \quad v^{(2)}=\left(\begin{array}{c}
s \xi_{1} \\
s \xi_{2} \\
-i \mu|\xi|^{2} \\
0
\end{array}\right)
$$

And for $\lambda_{3}$ we have the eigenvector

$$
v^{(3)}=\left(\begin{array}{c}
i \xi_{1} c \alpha\left(\alpha^{2}-\mu^{2}\right) \\
i \xi_{2} c \alpha\left(\alpha^{2}-\mu^{2}\right) \\
c \alpha\left(\alpha \sqrt{s^{2}+|\xi|^{2}\left(\alpha^{2}-\mu^{2}\right)}-s \mu\right) \\
\mu \sqrt{s^{2}+|\xi|^{2}\left(\alpha^{2}-\mu^{2}\right)}-s \alpha
\end{array}\right) .
$$

Eliminating $\psi$ from (5.5), we obtain

$$
\left\{\begin{array}{l}
\xi_{2} v_{1}-\xi_{1} v_{2}=0 \\
s \tau\left(\xi_{1} v_{1}+\xi_{2} v_{2}\right)-i|\xi|^{2}\left(\tau \mu v_{3}-k v_{4}\right)=0 \\
2 \nu v_{3}-c\left(\alpha^{2}+\mu^{2}\right) v_{4}=0
\end{array}\right.
$$

Applying the matrix on the left side of (5.8) to the three eigenvectors (5.6), (5.7), we have the matrix

$$
\left(\begin{array}{ccc}
|\xi|^{2} & 0 & 0 \\
0 & \tau|\xi|^{2}\left(s^{2}-\mu^{2}|\xi|^{2}\right) & -2 i \mu^{2}|\xi|^{2} \\
0 & 0 & c\left(\alpha^{2}-\mu^{2}\right)\left(\alpha s+\mu \sqrt{s^{2}+|\xi|^{2}\left(\alpha^{2}-\mu^{2}\right)}\right)
\end{array}\right)
$$


Since $s \neq \mu|\xi|,|\xi| \neq 0$, and $\alpha^{2}-\mu^{2}>0$, it follows that

$$
s^{2}-\mu^{2}|\xi|^{2} \neq 0, \quad \alpha s+\mu \sqrt{s^{2}+|\xi|^{2}\left(\alpha^{2}-\mu^{2}\right)} \neq 0 .
$$

Therefore, the matrix (5.9) is nonsingular.

Case II: $s \neq \mu|\xi|$ and $|\xi|=0$. In this case, we have for the eigenvalues $\lambda_{1}=\lambda_{2}$ and $\lambda_{3}$ three independent eigenvectors

$$
v^{(1)}=\left(\begin{array}{l}
1 \\
0 \\
0 \\
0
\end{array}\right), \quad v^{(2)}=\left(\begin{array}{l}
0 \\
1 \\
0 \\
0
\end{array}\right), \quad v^{(3)}=\left(\begin{array}{c}
0 \\
0 \\
c \alpha \\
-1
\end{array}\right) .
$$

Eliminating $\psi$ from (5.5), we have

$$
\left\{\begin{array}{l}
v_{1}=0 \\
v_{2}=0 \\
2 \nu v_{3}-c\left(\alpha^{2}+\mu^{2}\right) v_{4}=0 .
\end{array}\right.
$$

Applying the matrix on the left side of (5.11) to the three eigenvectors (5.10), we have the matrix

$$
\left(\begin{array}{ccc}
1 & 0 & 0 \\
0 & 1 & 0 \\
0 & 0 & c\left(\alpha^{2}+\mu^{2}\right)
\end{array}\right)
$$

which is obviously nonsingular.

Case III: $s=\mu|\xi|$. In this case, we have two eigenvectors $v^{(1)}, v^{(2)}$ for the eigenvalue $\lambda_{1}=\lambda_{2}=\lambda_{3}$ and one generalized eigenvector $v^{(3)}$ :

$$
v^{(1)}=\left(\begin{array}{c}
\xi_{2} \\
-\xi_{1} \\
0 \\
0
\end{array}\right), \quad v^{(2)}=\left(\begin{array}{c}
\xi_{1} \\
\xi_{2} \\
-i|\xi| \\
0
\end{array}\right), \quad v^{(3)}=\left(\begin{array}{c}
0 \\
0 \\
-k \\
\mu \tau
\end{array}\right) \text {. }
$$

Eliminating $\psi$ from (5.5), we have

$$
\left\{\begin{array}{l}
\xi_{2} v_{1}-\xi_{1} v_{2}=0 \\
s \tau\left(\xi_{1} v_{1}+\xi_{2} v_{2}\right)-i|\xi|^{2}\left(\tau \mu v_{3}-k v_{4}\right)=0 \\
2 \nu v_{3}-c\left(\alpha^{2}+\mu^{2}\right) v_{4}=0
\end{array}\right.
$$

Applying the matrix on the left side of (5.14) to the three vectors (5.13), we have the matrix

$$
\left(\begin{array}{ccc}
|\xi|^{2} & 0 & 0 \\
0 & 0 & -2 i \mu|\xi| \\
0 & i|\xi|^{2} \tau \mu k & 2 \mu c \tau\left(2 \alpha^{2}+\mu^{2}\right)
\end{array}\right)
$$

which is also obviously nonsingular.

This completes the proof of Proposition 3.1. 


\section{REFERENCES}

[1] M. Beals and M. C. Reed, Microlocal regularity for nonsmooth pseudo-differential operators and applications to nonlinear problems, Trans. Amer. Math. Soc. 285, 159-184 (1984)

[2] A. T. Bui and D. Li, The double shock front solutions for hyperbolic conservation laws in multidimensional space, Trans. Amer. Math. Soc. 316, 233-250 (1989)

[3] A. Friedman and B. Hu, The Stefan problem for a hyperbolic heat equation, IMA preprint, no. 348

[4] J. Greenberg, A hyperbolic heat transfer problem with phase changes, IMA J. Appl. Math. 38, 1-21 (1987)

[5] D. D. Joseph and Luigi Preziosi, Heat waves, Rev. Modern Phys. 61, 41-73 (1989)

[6] H.-O. Kreiss, Initial boundary value problems for hyperbolic systems, Comm. Pure Appl. Math. 23, 277-298 (1970)

[7] D. Li, Nonlinear initial-boundary value problem and the existence on multi-D shock wave for quasilinear hyperbolic-parabolic coupled systems, Chinese Ann. Math. Ser. B 8, 252-280 (1987)

[8] D. Li, The well-posedness of a hyperbolic Stefan problem, Quart. Appl. Math. 47, 221-231 (1989)

[9] D. Li, A one-phase hyperbolic Stefan problem in multi-dimensional space, Trans. Amer. Math. Soc. 318, 401-415 (1990)

[10] A. Majda, The stability of multi-dimensional shock fronts, Mem. Amer. Math. Soc., No. 275 (1983)

[11] A. Majda, The existence of multi-dimensional shock fronts, Mem. Amer. Math. Soc., No. 281 (1983)

[12] J. Rauch and F. Massey, Differentiability of solutions to hyperbolic initial-boundary value problems, Trans. Amer. Math. Soc. 189, 303-318 (1974)

[13] R. E. Showalter and N. J. Walkington, A hyperbolic Stefan problem, Quart. Appl. Math. 45, 769$782(1987)$

[14] A. Solomon, V. Alexiades, D. Wilson, and J. Drake, On the formulation of a hyperbolic Stefan problem, Quart. Appl. Math. 43, 295-304 (1985)

[15] A. Solomon, V. Alexiades, D. Wilson, and J. Greenberg, A hyperbolic Stefan problem with discontinuous temperature, ORNL-6216, March, 1986 